\title{
An Intrinsic Characterization of Bonnet Surfaces Based on a Closed Differential Ideal
}

\author{
Paul Bracken \\ Department of Mathematics, University of Texas, Edinburg, TX 78540-2999, USA \\ Correspondence should be addressed to Paul Bracken; bracken@utpa.edu
}

Received 27 May 2014; Accepted 14 August 2014; Published 31 August 2014

Academic Editor: Chandrashekar Devchand

Copyright (C) 2014 Paul Bracken. This is an open access article distributed under the Creative Commons Attribution License, which permits unrestricted use, distribution, and reproduction in any medium, provided the original work is properly cited.

The structure equations for a two-dimensional manifold are introduced and two results based on the Codazzi equations pertinent to the study of isometric surfaces are obtained from them. Important theorems pertaining to isometric surfaces are stated and a theorem due to Bonnet is obtained. A transformation for the connection forms is developed. It is proved that the angle of deformation must be harmonic, and that the differentials of many of the important variables generate a closed differential ideal. This implies that a coordinate system exists in which many of the variables satisfy particular ordinary differential equations, and these results can be used to characterize Bonnet surfaces.

\section{Introduction}

Bonnet surfaces in three-dimensional Euclidean space have been of great interest for a number of reasons as a type of surface $[1,2]$ for a long time. Bonnet surfaces are of nonconstant mean curvature that admit infinitely many nontrivial and geometrically distinct isometries which preserve the mean curvature function. Nontrivial isometries are ones that do not extend to isometries of the whole space $E^{3}$. Considerable interest has resulted from the fact that the differential equations that describe the Gauss equations are classified by the type of related Painleve equations they correspond to and they are integrated in terms of certain hypergeometric transcendents [3-5]. Here the approach first given by Chern [6] to Bonnet surfaces is considered. The developement is accessible with many new proofs given. The main intention is to end by deriving an intrinsic characterization of these surfaces which indicates they are analytic. Moreover, it is shown that a type of Lax pair can be given for these surfaces and integrated. Several of the more important functions such as the mean curvature are seen to satisfy nontrivial ordinary differential equations.

Quite a lot is known about these surfaces. With many results the analysis is local and takes place under the assumptions that the surfaces contain no umbilic points and no critical points of the mean curvature function. The approach here allows the elimination of many assumptions and it is found that the results are not too different from the known local ones. The statements and proofs have been given in great detail in order to help illustrate and display the interconnectedness of the ideas and results involved.

To establish some information about what is known, consider an oriented, connected, smooth open surface $M$ in $E^{3}$ with nonconstant mean curvature function $H$. Moreover, $M$ admits infinitely many nontrivial and geometrically distinct isometries preserving $H$. Suppose that $U$ is the set of umbilic points of $M$ and $V$ is the set of critical points of $H$. Many global facts are known with regard to $U, V$, and $H$, and a few will now be mentioned. The set $U$ consists of isolated points, even if there exists only one nontrivial isometry preserving the mean curvature; moreover, $U \subset V[7,8]$. Interestingly, there is no point in $V-U$ at which all order derivatives of $H$ are zero, and $V$ cannot contain any curve segment. If the function by which a nontrivial isometry preserving the mean curvature rotates the principal frame is considered, as when there are infinitely many isometries, this function is a global function on $M$ continuously defined [9-11]. As first noted by Chern [6], this function is harmonic. The analysis will begin by formulating the structure equations for two-dimensional manifolds. 


\section{Structure Equations}

Over $M$ there exists a well defined field of orthonormal frames which is written as $\mathbf{x}, e_{1}, e_{2}, e_{3}$ such that $x \in M, e_{3}$ is the unit normal at $\mathbf{x}$ and $e_{1}, e_{2}$ are along principal directions [12]. The fundamental equations for $M$ have the form

$$
\begin{gathered}
d \mathbf{x}=\omega_{1} e_{1}+\omega_{2} e_{2}, \quad d e_{1}=\omega_{12} e_{2}+\omega_{13} e_{3}, \\
d e_{2}=-\omega_{12} e_{1}+\omega_{23} e_{3}, \quad d e_{3}=-\omega_{13} e_{1}-\omega_{23} e_{2} .
\end{gathered}
$$

Differentiating each of these equations in turn, results in a large system of equations for the exterior derivatives of the $\omega_{i}$ and the $\omega_{i j}$, as well as a final equation which relates some of the forms [13]. This choice of frame and Cartan's lemma allows for the introduction of the two principal curvatures which are denoted by $a$ and $c$ at $\mathbf{x}$ by writing

$$
\omega_{12}=h \omega_{1}+k \omega_{2}, \quad \omega_{13}=a \omega_{1}, \quad \omega_{23}=c \omega_{2} .
$$

Suppose that $a>c$ in the following. The mean curvature of $M$ is denoted by $H$ and the Gaussian curvature by $K$. They are related to $a$ and $c$ as follows:

$$
H=\frac{1}{2}(a+c), \quad K=a \cdot c .
$$

The forms which appear in (1) satisfy the fundamental structure equations which are summarized here [14],

$$
\begin{gathered}
d \omega_{1}=\omega_{12} \wedge \omega_{2}, \quad d \omega_{2}=\omega_{1} \wedge \omega_{12}, \\
d \omega_{13}=\omega_{12} \wedge \omega_{23}, \quad d \omega_{23}=\omega_{13} \wedge \omega_{12}, \\
d \omega_{12}=a c \omega_{2} \wedge \omega_{1}=-K \omega_{1} \wedge \omega_{2} .
\end{gathered}
$$

The second pair of equations of (4) is referred to as the Codazzi equation and the last equation is the Gauss equation.

Exterior differentiation of the two Codazzi equations yields

$$
\begin{aligned}
& \left(d a-(a-c) h \omega_{2}\right) \wedge \omega_{1}=0 \\
& \left(d c-(a-c) k \omega_{1}\right) \wedge \omega_{2}=0 .
\end{aligned}
$$

Cartan's lemma can be applied to the equations in (5). Thus there exist two functions $u$ and $v$ such that

$$
\begin{aligned}
& \frac{1}{a-c} d a-h \omega_{2}=(u-k) \omega_{1}, \\
& \frac{1}{a-c} d c-k \omega_{1}=(v-h) \omega_{2} .
\end{aligned}
$$

Subtracting the pair of equations in (6) gives an expression for $d \log (a-c)$

$$
d \log (a-c)=(u-2 k) \omega_{1}-(v-2 h) \omega_{2} .
$$

Define the variable $J$ to be

$$
J=\frac{1}{2}(a-c)>0 .
$$

It will appear frequently in what follows. Equation (7) then takes the form

$$
d \log J=(u-2 k) \omega_{1}-(v-2 h) \omega_{2} .
$$

The $\omega_{i}$ constitute a linearly independent set. Two related coframes called $\vartheta_{i}$ and $\alpha_{i}$ can be defined in terms of the $\omega_{i}$ and the functions $u$ and $v$ as follows:

$$
\begin{array}{ll}
\vartheta_{1}=u \omega_{1}+v \omega_{2}, & \vartheta_{2}=-v \omega_{1}+u \omega_{2}, \\
\alpha_{1}=u \omega_{1}-v \omega_{2}, & \alpha_{2}=v \omega_{1}+u \omega_{2} .
\end{array}
$$

These relations imply that $\vartheta_{1}=0$ is tangent to the level curves specified by $H$ equals constant and $\alpha_{1}=0$ is its symmetry with respect to the principal directions.

Squaring both sides of the relation $2 H=a+c$ and subtracting the relation $4 K=4 a c$ yields $4\left(H^{2}-K\right)=(a-c)^{2}$. The Hodge operator, denoted by $*$, will play an important role throughout. It produces the following result on the basis forms $\omega_{i}$,

$$
* \omega_{1}=\omega_{2}, \quad * \omega_{2}=-\omega_{1}, \quad *^{2}=-1 .
$$

Moreover, adding the expressions for $d a$ and $d c$ given in (6), and the results are

$$
\begin{aligned}
\frac{1}{a-c}(d a+d c) & =(u-k) \omega_{1}+h \omega_{2}+(v-k) \omega_{2}+k \omega_{1} \\
& =u \omega_{1}+v \omega_{2}=\vartheta_{1} .
\end{aligned}
$$

Finally, note that

$$
\begin{aligned}
\alpha_{1}+2 * \omega_{12} & =u \omega_{1}-v \omega_{2}+2 *\left(h \omega_{1}+k \omega_{2}\right) \\
& =(u-2 k) \omega_{1}-(v-2 h) \omega_{2}=d \log J
\end{aligned}
$$

Therefore, the Codazzi equations (12) and (13) can be summarized using the definitions of $H$ and $J$ as

$$
d H=J \vartheta_{1}, \quad d \log J=\alpha_{1}+2 * \omega_{12} .
$$

\section{A Theorem of Bonnet}

Suppose that $M^{*}$ is a surface which is isometric to $M$ such that the principal curvatures are preserved [10-12]. Denote all quantities which pertain to $M^{*}$ with the same symbols but with asterisks, as for example,

$$
a^{*}=a, \quad c^{*}=c .
$$

The same notation will be applied to the variables and forms which pertain to $M$ and $M^{*}$. When $M$ and $M^{*}$ are isometric, the forms $\omega_{i}$ are related to the $\omega_{i}^{*}$ by the following transformation

$$
\omega_{1}^{*}=\cos \tau \omega_{1}-\sin \tau \omega_{2}, \quad \omega_{2}^{*}=\sin \tau \omega_{1}+\cos \tau \omega_{2} .
$$

Theorem 1. Under the transformation of coframe given by (16), the associated connection forms are related by

$$
\omega_{12}^{*}=\omega_{12}-d \tau .
$$


Proof. Exterior differentiation of $\omega_{1}^{*}$ produces

$$
\begin{aligned}
d \omega_{1}^{*}= & -\sin \tau d \tau \wedge \omega_{1}+\cos \tau d \omega_{1}-\cos \tau d \tau \wedge \omega_{2} \\
& -\sin \tau d \omega_{2} \\
= & d \tau \wedge\left(-\sin \tau \omega_{1}-\cos \tau \omega_{2}\right)+\cos \tau \omega_{12} \wedge \omega_{2} \\
& -\sin \tau \omega_{1} \wedge \omega_{12}=\left(-d \tau+\omega_{12}\right) \wedge \omega_{2}^{*} .
\end{aligned}
$$

Similarly, differentiating $\omega_{2}^{*}$ gives

$$
\begin{aligned}
d \omega_{2}^{*}= & \cos \tau \wedge \omega_{1}+\sin \tau d \omega_{1}-\sin \tau d \tau \wedge \omega_{2}+\cos \tau d \omega_{2} \\
= & d \tau \wedge\left(\cos \tau \omega_{1}-\sin \tau \omega_{2}\right)+\sin \tau \omega_{12} \wedge \omega_{2} \\
& +\cos \tau \omega_{1} \wedge \omega_{12}=\omega_{1}^{*} \wedge\left(-d \tau+\omega_{12}\right) .
\end{aligned}
$$

There is a very important result which can be developed at this point. In the case that $a=a^{*}$ and $c=c^{*}$, the Codazzi equations imply that

$$
\begin{aligned}
\alpha_{1}+2 * \omega_{12} & =d \log (a-c) \\
& =d \log \left(a^{*}-c^{*}\right) \\
& =\alpha_{1}^{*}+2 * \omega_{12}^{*} .
\end{aligned}
$$

Apply the operator $*$ to both sides of this equation, we obtain

$$
\alpha_{2}-2 \omega_{12}=\alpha_{2}^{*}-2 \omega_{12}^{*} \text {. }
$$

Substituting for $\omega_{12}^{*}$ from Theorem 1 , this is

$$
2 d \tau=\alpha_{2}-\alpha_{2}^{*}
$$

Lemma 2. Consider

$$
\vartheta_{1}=\vartheta_{1}^{*}
$$

Proof. This can be shown in two ways. First from (16), express the $\omega_{i}$ in terms of the $\omega_{i}^{*}$ to give

$$
\omega_{1}=\cos \tau \omega_{1}^{*}+\sin \tau \omega_{2}^{*}, \quad \omega_{2}=-\sin \tau \omega_{1}^{*}+\cos \tau \omega_{2}^{*} \text {. }
$$

Therefore,

$$
\begin{aligned}
\vartheta_{1}= & u \omega_{1}+v \omega_{2} \\
= & u\left(\cos \tau \omega_{1}^{*}+\sin \tau \omega_{2}^{*}\right) \\
& +v\left(-\sin \tau \omega_{1}^{*}+\cos \tau \omega_{2}^{*}\right) \\
= & u^{*} \omega_{1}^{*}+v^{*} \omega_{2}^{*}=\vartheta_{1}^{*},
\end{aligned}
$$

where $u^{*}=u \cos \tau-v \sin \tau$ and $v^{*}=u \sin \tau+v \cos \tau$. (8).

Lemma 2 also follows from the fact that $d H=d H^{*}$ and
Lemma 3. Consider

$$
\alpha_{2}^{*}=\sin (2 \tau) \alpha_{1}+\cos (2 \tau) \alpha_{2} .
$$

Proof.

$$
\begin{aligned}
\alpha_{2}^{*}= & (u \sin \tau+v \cos \tau)\left(\cos \tau \omega_{1}-\sin \tau \omega_{2}\right) \\
& +(u \cos \tau-v \sin \tau)\left(\sin \tau \omega_{1}+\cos \tau \omega_{2}\right) \\
= & (u \sin (2 \tau)+v \cos (2 \tau)) \omega_{1} \\
& +(-v \sin (2 \tau)+u \cos (2 \tau)) \omega_{2} \\
= & \sin (2 \tau) \alpha_{1}+\cos (2 \tau) \alpha_{2} .
\end{aligned}
$$

Substituting $\alpha_{2}^{*}$ from Lemma 3 into (22), $d \tau$ can be written as

$$
\begin{aligned}
d \tau & =\frac{1}{2}\left(\alpha_{2}-\sin (2 \tau) \alpha_{1}-\cos (2 \tau) \alpha_{2}\right) \\
& =\frac{1}{2}\left((1-\cos (2 \tau)) \alpha_{2}-\sin (2 \tau) \alpha_{1}\right) .
\end{aligned}
$$

Introduce the new variable $t=\cot (\tau)$ so $d t=-\csc ^{2}(\tau) d \tau$ and $\sin \tau=1 / \sqrt{1+t^{2}}, \cos \tau=1 / \sqrt{1+t^{2}}$, hence the following lemma.

Lemma 4. Consider

$$
d t=t \alpha_{1}-\alpha_{2}
$$

This is the total differential equation which must be satisfied by the angle $\tau$ of rotation of the principal directions during the deformation. If the deformation is to be nontrivial, it must be that this equation is completely integrable.

Theorem 5. A surface $M$ admits a nontrivial isometric deformation that keeps the principal curvatures fixed if and only if

$$
d \alpha_{1}=0, \quad d \alpha_{2}=\alpha_{1} \wedge \alpha_{2}
$$

or $\alpha_{12}=\alpha_{2}$.

Proof. Differentiating both sides of Lemma 4 gives

$$
d t \wedge \alpha_{1}+t d \alpha_{1}-d \alpha_{2}=\left(t \alpha_{1}-\alpha_{2}\right) \wedge \alpha_{1}+t d \alpha_{1}-d \alpha_{2}=0
$$

Equating the coefficients of $t$ to zero gives the result (30).

This theorem seems to originate with Chern [6] and is very useful because it gives the exterior derivatives of the $\alpha_{i}$. When the mean curvature is constant, $d H=0$, hence it follows from (14) that $\vartheta_{1}=0$. This implies that $u=v=0$, and so $\alpha_{1}$ and $\alpha_{2}$ must vanish. Hence $d t=0$ which implies that, since the $\alpha_{i}$ are linearly independent, $t$ equals a constant. Thus, we arrive at a theorem originally due to Bonnet.

Theorem 6. A surface of constant mean curvature can be isometrically deformed preserving the principal curvatures. During the deformation, the principal directions rotate by a fixed angle. 


\section{Connection Form Associated to a Coframe and Transformation Properties}

Given the linearly independent one-forms $\omega_{1}, \omega_{2}$, the first two of the structure equations uniquely determine the form $\omega_{12}$. The $\omega_{1}, \omega_{2}$ are called the orthonormal coframe of the metric

$$
d s^{2}=\omega_{1}^{2}+\omega_{2}^{2}
$$

and $\omega_{12}$ the connection form associated with it.

Theorem 7. Suppose that $A>0$ is a function on $M$. Under the change of coframe,

$$
\omega_{1}^{*}=A \omega_{1}, \quad \omega_{2}^{*}=A \omega_{2},
$$

the associated connection forms are related by

$$
\omega_{12}^{*}=\omega_{12}+* d \log A \text {. }
$$

Proof. The structure equations for the transformed system are given as

$$
d \omega_{1}^{*}=\omega_{12}^{*} \wedge \omega_{2}^{*}, \quad d \omega_{2}^{*}=\omega_{1}^{*} \wedge \omega_{12}^{*} .
$$

Using (33) to replace the $\omega_{i}^{*}$ in these, we obtain

$$
\begin{aligned}
& d \log A \wedge \omega_{1}+d \omega_{1}=\omega_{12}^{*} \wedge \omega_{2} \\
& d \log A \wedge \omega_{2}+d \omega_{2}=\omega_{1} \wedge \omega_{12}^{*}
\end{aligned}
$$

The set of $\omega_{i}$ satisfies a similar system of structure equations, so replacing $d \omega_{i}$ here yields

$$
\begin{aligned}
& \left(\omega_{12}^{*}-\omega_{12}\right) \wedge \omega_{2}=d \log A \wedge \omega_{1}, \\
& \left(\omega_{12}^{*}-\omega_{12}\right) \wedge \omega_{1}=-d \log A \wedge \omega_{2} .
\end{aligned}
$$

Since the forms $\omega_{i}$ satisfy the equations $* \omega_{1}=\omega_{2}$ and $* \omega_{2}=$ $-\omega_{1}$, substituting these relations into the above equations and using $\Omega_{k} \wedge\left(* \Theta_{k}\right)=\Theta_{k} \wedge\left(* \Omega_{k}\right)$, we obtain that in the form

$$
\begin{aligned}
& \omega_{1} \wedge *\left(\omega_{12}^{*}-\omega_{12}\right)=-\omega_{1} \wedge d \log A, \\
& \omega_{2} \wedge *\left(\omega_{12}^{*}-\omega_{12}\right)=-\omega_{2} \wedge d \log A .
\end{aligned}
$$

Cartan's lemma can be used to conclude from these that there exist functions $f$ and $g$ such that

$$
\begin{aligned}
& *\left(\omega_{12}^{*}-\omega_{12}\right)=-d \log A-f \omega_{1}, \\
& *\left(\omega_{12}^{*}-\omega_{12}\right)=-d \log A+g \omega_{2} .
\end{aligned}
$$

Finally, apply $*$ to both sides and use $*^{2}=-1$ to obtain

$$
\begin{aligned}
& \omega_{12}^{*}-\omega_{12}=* d \log A+f \omega_{2}, \\
& \omega_{12}^{*}-\omega_{12}=* d \log A+g \omega_{1} .
\end{aligned}
$$

The forms $\omega_{i}$ are linearly independent, so for these two equations to be compatible, it suffices to put $f=g=0$, and the result follows.
For the necessity in the Chern criterion, Theorem 5, no mention of the set $V$ of critical points of $H$ is needed. In fact, when $H$ is constant, this criterion is met and the sufficiency also holds with $\tau$ constant. However, when $H$ is not identically constant, we need to take the set $V$ of critical points into account for the sufficiency. In this case, $M-V$ is also an open, dense, and connected subset of $M$. On this subset $J>0$ and the function $A$ can be defined in terms of the functions $u$ and $v$ as

$$
A=+\sqrt{u^{2}+v^{2}}>0 .
$$

To define more general transformations of the $\omega_{i}$, define the angle $\psi$ as

$$
u=A \cos (\psi), \quad v=A \sin (\psi) .
$$

This angle which is defined modulo $2 \pi$, is continuous only locally and could be discontinuous in a nonsimply connected region of $M-V$. With $A$ and $\psi$ related to $u$ and $v$ by (42), the forms $\vartheta_{i}$ and $\alpha_{i}$ can be written in terms of $A$ and $\psi$ as

$$
\begin{aligned}
& \vartheta_{1}=A\left(\cos (\psi) \omega_{1}+\sin (\psi) \omega_{2}\right), \\
& \vartheta_{2}=A\left(-\sin (\psi) \omega_{1}+\cos (\psi) \omega_{2}\right), \\
& \alpha_{1}=A\left(\cos (\psi) \omega_{1}-\sin (\psi) \omega_{2}\right), \\
& \alpha_{2}=A\left(\sin (\psi) \omega_{1}+\cos (\psi) \omega_{2}\right) .
\end{aligned}
$$

The forms $\omega_{i}, \vartheta_{i}$, and $\alpha_{i}$ define the same structure on $M$ and we let $\omega_{12}, \vartheta_{12}$, and $\alpha_{12}$ be the connection forms associated to the coframes $\omega_{1}, \omega_{2} ; \vartheta_{1}, \vartheta_{2} ; \alpha_{1}, \alpha_{2}$. The next Theorem is crucial for what follows.

\section{Theorem 8.}

$$
\vartheta_{12}=d \psi+\omega_{12}+* d \log A=2 d \psi+\alpha_{12} .
$$

Proof. Each of the transformations which yield the $\vartheta_{i}$ and $\alpha_{i}$ in the form (43) can be thought of as a composition of the two transformations which occur in the Theorems 1 and 7. First apply the transformation $\omega_{i} \rightarrow A \omega_{i}$ and $\tau \rightarrow-\psi$ with $\omega_{i}^{*} \rightarrow \vartheta_{i}$ in (16), we get the $\vartheta_{i}$ equations in (43). Invoking Theorems 1 and 7 in turn, the first result is obtained

$$
\vartheta_{12}=d \psi+\omega_{12}+* d \log A \text {. }
$$

The transformation to the $\alpha_{i}$ is exactly similar except that $\tau \rightarrow \psi$, hence

$$
\alpha_{12}=-d \psi+\omega_{12}+* d \log A
$$

This implies that $* d \log A=\alpha_{12}+d \psi-\omega_{12}$. When replaced in the first equation of (44), the second equation appears. Note that from Theorem $5, \alpha_{12}=\alpha_{2}$, so the second equation can be given as $\vartheta_{12}=2 d \psi+\alpha_{2}$.

Differentiating the second equation in (14) and using $d \alpha_{1}=0$, it follows that

$$
d * \omega_{12}=0
$$


Lemma 9. The angle $\psi$ is a harmonic function $d * d \psi=0$ and moreover, $d * \vartheta_{12}=0$.

Proof. From Theorem 8, it follows by applying $*$ through (44) that

$$
* \vartheta_{12}=* \omega_{12}+* d \psi-d \log A=2 * d \psi-\alpha_{1} .
$$

Exterior differentiation of this equation using $d * \omega_{12}=0$ immediately gives

$$
d * d \psi=0
$$

This states that $\psi$ is a harmonic function. Equation (48) also implies that $d * \vartheta_{12}=0$.

\section{Construction of the Closed Differential Ideal Associated with $M$}

Exterior differentiation of the first equation in (14) and using the second equation produces

$$
d \vartheta_{1}+\left(\alpha_{1}+2 * \omega_{12}\right) \wedge \vartheta_{1}=0 .
$$

The structure equation for the $\vartheta_{i}$ will be needed,

$$
d \vartheta_{1}=\vartheta_{12} \wedge \vartheta_{2}=-* \vartheta_{12} \wedge \vartheta_{1} .
$$

From the second equation in (44), we have $* \omega_{12}-d \log A+$ $\alpha_{1}=* d \psi$, and putting this in the first equation of (44) we find

$$
-* \vartheta_{12}+\alpha_{1}+2 * \omega_{12}=2 d \log A .
$$

Using (52) in (51),

$$
d \vartheta_{1}+\left(\alpha_{1}+2 * \omega_{12}\right) \wedge \vartheta_{1}=2 d \log A \wedge \vartheta_{1} .
$$

Replacing $d \vartheta_{1}$ by means of (50) implies the following important result

$$
d \log A \wedge \vartheta_{1}=0
$$

Equation (54) and Cartan's lemma imply that there exists a function $B$ such that

$$
d \log A=B \vartheta_{1} .
$$

This is the first in a series of results which relates many of the variables in question such as $J, B$, and $\vartheta_{12}$ directly to the oneform $\vartheta_{1}$. To show this requires considerable work. The way to proceed is to use the forms $\alpha_{i}$ in Theorem 5 because their exterior derivatives are known. For an arbitrary function on $M$, define

$$
d f=f_{1} \alpha_{1}+f_{2} \alpha_{2}
$$

Differentiating (56) and extracting the coefficient of $\alpha_{1} \wedge \alpha_{2}$, we obtain

$$
f_{21}-f_{12}+f_{2}=0
$$

In terms of the $\alpha_{i}, * d \psi=\psi_{1} \alpha_{2}-\psi_{2} \alpha_{1}$, Lemma 9 yields

$$
\psi_{11}+\psi_{22}+\psi_{1}=0
$$

Finally, since $* \vartheta_{12}=2 * d \psi-\alpha_{1}$, substituting for $* d \psi$, we obtain that

$$
* \vartheta_{12}=-\left(2 \psi_{2}+1\right) \alpha_{1}+2 \psi_{1} \alpha_{2} \text {. }
$$

Differentiating structure equation (51) and using Lemma 9,

$$
* \vartheta_{12} \wedge d \vartheta_{1}=0
$$

so,

$$
* \vartheta_{12} \wedge \vartheta_{12} \wedge \vartheta_{2}=0 .
$$

This equation implies that either $\vartheta_{12}$ or $* \vartheta_{12}$ is a multiple by a function of the form $\vartheta_{2}$. Hence, for some function $p$,

$$
\begin{array}{ll}
\vartheta_{12}=-p \vartheta_{2}, & * \vartheta_{12}=p \vartheta_{1}, \\
\vartheta_{12}=p \vartheta_{1}, & * \vartheta_{12}=p \vartheta_{2} .
\end{array}
$$

Substituting the first line of (62) back into the structure equation, we have

$$
d \vartheta_{1}=0 .
$$

The second line yields simply $d \vartheta_{1}=p \vartheta_{1} \wedge \vartheta_{2}$. Only the first case is examined now. Substituting (63) into (50), the following important constraint is obtained

$$
\left(\alpha_{1}+2 * \omega_{12}\right) \wedge \vartheta_{1}=0 .
$$

Theorem 10. The function $\psi$ satisfies the equation

$$
2 \psi_{1} \cos (2 \psi)+\left(2 \psi_{2}+1\right) \sin (2 \psi)=0
$$

Proof. By substituting $* d \psi$ into (48) we have

$$
* \vartheta_{12}=2 *\left(\psi_{1} \alpha_{1}+\psi_{2} \alpha_{2}\right)-\alpha_{1}=-\left(2 \psi_{2}+1\right) \alpha_{1}+2 \psi_{1} \alpha_{2} .
$$

Substituting (66) into (44) and solving for $* \omega_{12}$, we obtain that

$$
\begin{aligned}
* \omega_{12} & =* \vartheta_{12}-* d \psi+d \log A=* \vartheta_{12}-* d \psi+B \vartheta_{1} \\
& =* d \psi-\alpha_{1}+B \vartheta_{1} .
\end{aligned}
$$

This can be put in the equivalent form

$$
2 * \omega_{12}+\alpha_{1}=2 * d \psi-\alpha_{1}+2 B \vartheta_{1} .
$$

Taking the exterior product with $\vartheta_{1}$ and using $d \psi_{1}$, we get

$$
\begin{aligned}
& \left(\alpha_{1}+2 * \omega_{12}\right) \wedge \vartheta_{1} \\
& \quad=\left(2 * d \psi-\alpha_{1}\right) \wedge \vartheta_{1}=\left(2 \psi_{1} * \alpha_{1}+2 \psi_{2} * \alpha_{2}-\alpha_{1}\right) \wedge \vartheta_{1} \\
& \quad=\left(2 \psi_{1} \cos (2 \psi)+\left(2 \psi_{2}+1\right) \sin (2 \psi)\right) \vartheta_{2} \wedge \vartheta_{1} .
\end{aligned}
$$

Imposing the constraint (64), the coefficient of $\vartheta_{1} \wedge \vartheta_{2}$ can be equated to zero. This produces the result (65). 
As a consequence of Theorem 10, a new function $C$ can be introduced such that

$$
2 \psi_{1}=C \sin (2 \psi), \quad 2 \psi_{2}+1=-C \cos (2 \psi) .
$$

Differentiating each of these with respect to the $\alpha_{i}$ basis, we get for $i=1,2$ that

$$
\begin{aligned}
& 2 \psi_{1 i}=C_{i} \sin (2 \psi)+2 \psi_{i} C \cos (2 \psi), \\
& 2 \psi_{2 i}=-C_{i} \cos (2 \psi)+2 \psi_{i} C \sin (2 \psi) .
\end{aligned}
$$

Substituting $f=\psi$ into (57) and using the fact that $\psi$ satisfies (58) gives the pair of equations

$$
\begin{gathered}
-C_{1} \cos (2 \psi)-C_{2} \sin (2 \psi)+2 \psi_{1} C \sin (2 \psi) \\
-\left(2 \psi_{2}+1\right) C \cos (2 \psi)-1=0, \\
C_{1} \sin (2 \psi)-C_{2} \cos (2 \psi)+2 \psi_{1} C \cos (2 \psi) \\
+\left(2 \psi_{2}+1\right) C \sin (2 \psi)=0 .
\end{gathered}
$$

This linear system can be solved for $C_{1}$ and $C_{2}$ to get

$$
\begin{gathered}
C_{1}+C\left(2 \psi_{2}+1\right)+\cos (2 \psi)=0, \\
C_{2}-2 C \psi_{1}+\sin (2 \psi)=0 .
\end{gathered}
$$

By differentiating each of the equations in (73), it is easy to verify that $C$ satisfies (57), namely, $C_{12}-C_{21}-C_{2}=0$. Hence there exist harmonic functions which satisfy (65). The solution depends on two arbitrary constants, the values of $\psi$ and $C$ at an initial point.

\section{Lemma 11. Consider}

$$
d C=\left(C^{2}-1\right) \vartheta_{1}, \quad * \vartheta_{12}=C \vartheta_{1} .
$$

Proof. It is easy to express the $\vartheta_{i}$ in terms of the $\alpha_{i}$,

$$
\begin{aligned}
& \vartheta_{1}=\cos (2 \psi) \alpha_{1}+\sin (2 \psi) \alpha_{2}, \\
& \vartheta_{2}=-\sin (2 \psi) \alpha_{1}+\cos (2 \psi) \alpha_{2} .
\end{aligned}
$$

Therefore, using (70) and (73), it is easy to see that

$$
\begin{aligned}
d C & =C_{1} \alpha_{1}+C_{2} \alpha_{2}=\left(C^{2}-1\right)\left(\cos (2 \psi) \alpha_{1}+\sin (2 \psi) \alpha_{2}\right) \\
& =\left(C^{2}-1\right) \vartheta_{1} .
\end{aligned}
$$

Using (70), it follows that

$$
\begin{aligned}
* \vartheta_{12} & =-\left(2 \psi_{2}+1\right) \alpha_{1}+2 \psi_{1} \alpha_{2} \\
& =C \cos (2 \psi) \alpha_{1}+C \sin (2 \psi) \alpha_{2} \\
& =C\left(\cos (2 \psi) \alpha_{1}+\sin (2 \psi) \alpha_{2}\right)=C \vartheta_{1} .
\end{aligned}
$$

This implies that $\vartheta_{12}=-C \vartheta_{2}$.
It is possible to obtain formulas for $B_{1}, B_{2}$. Using (75) in (55), the derivatives of $\log A$ can be written down

$$
(\log A)_{1}=B \cos (2 \psi), \quad(\log A)_{2}=B \sin (2 \psi) .
$$

Differentiating each of these in turn, we obtain for $i=1,2$,

$$
\begin{aligned}
& (\log A)_{1 i}=B_{i} \cos (2 \psi)-2 B \psi_{i} \sin (2 \psi), \\
& (\log A)_{2 i}=B_{i} \sin (2 \psi)+2 B \psi_{i} \cos (2 \psi) .
\end{aligned}
$$

Taking $f=\log A$ in (57) produces a first equation for the $B_{i}$,

$$
\begin{aligned}
& B_{1} \sin (2 \psi)+2 B \psi_{1} \cos (2 \psi)-B_{2} \cos (2 \psi)+2 B \psi_{2} \sin (2 \psi) \\
& \quad+B \sin (2 \psi)=0 .
\end{aligned}
$$

If another equation in terms of $B_{1}$ and $B_{2}$ can be found, it can be solved simultaneously with (80). There exists such an equation and it can be obtained from the Gauss equation in (4) which we put in the form

$$
d \omega_{12}=-a c \omega_{1} \wedge \omega_{2}=-a c A^{-2} \alpha_{1} \wedge \alpha_{2}
$$

Solving (44) for $\omega_{12}$, we have

$$
\omega_{12}=d \psi+\alpha_{2}+(\log A)_{2} \alpha_{1}-(\log A)_{1} \alpha_{2} .
$$

The exterior derivative of this takes the form,

$$
d \omega_{12}=\left[1-(\log A)_{11}-(\log A)_{22}-(\log A)_{1}\right] \alpha_{1} \wedge \alpha_{2} .
$$

Putting this in the Gauss equation,

$$
-(\log A)_{11}-(\log A)_{22}+\left\{-(\log A)_{1}+1\right\}+a c A^{-2}=0 \text {. }
$$

Replacing the second derivatives from (79), we have the required second equation

$$
\begin{aligned}
- & B_{1} \cos (2 \psi)-B_{2} \sin (2 \psi) \\
& +B\left\{2 \psi_{1} \sin (2 \psi)-\left(2 \psi_{2}+1\right) \cos (2 \psi)\right\}+1+a c A^{-2}=0 .
\end{aligned}
$$

Solving (80) and (85) together, the following expressions for $B_{1}$ and $B_{2}$ are obtained

$$
\begin{gathered}
B_{1}+B\left(2 \psi_{2}+1\right)-\left(1+a c A^{-2}\right) \cos (2 \psi)=0, \\
B_{2}-2 B \psi_{1}-\left(1+a c A^{-2}\right) \sin (2 \psi)=0 .
\end{gathered}
$$

Given these results for $B_{1}$ and $B_{2}$, it is easy to produce the following two Lemmas.

\section{Lemma 12. Consider}

$$
d B=\left(B C+1+a c A^{-2}\right) \vartheta_{1}, \quad d \log J=(C+2 B) \vartheta_{1} .
$$


Proof. Substituting (86) into $d B$, we get

$$
\begin{aligned}
d B & =B_{1} \alpha_{1}+B_{2} \alpha_{2} \\
& =\left(B C+1+a c A^{-2}\right)\left(\cos (2 \psi) \alpha_{1}+\sin (2 \psi) \alpha_{2}\right) \\
& =\left(B C+1+a c A^{-2}\right) \vartheta_{1} .
\end{aligned}
$$

Moreover,

$$
\begin{aligned}
d \log J & =\alpha_{1}+2 * \omega_{12}=\alpha_{1}+2\left(* \vartheta_{12}-* d \psi+d \log A\right) \\
& =\alpha_{1}+2 * \vartheta_{12}-2 * d \psi+2 d \log A \\
& =* \vartheta_{12}+2 d \log A=C \vartheta_{1}+2 B \vartheta_{1} .
\end{aligned}
$$

Lemma 13. Consider

$$
d \psi=-\frac{1}{2} \sin (2 \psi) \vartheta_{1}-\frac{1}{2}(C+\cos (2 \psi)) \vartheta_{2} .
$$

Proof. Consider

$$
\begin{aligned}
2 d \psi= & 2 \psi_{1} \alpha_{1}+2 \psi_{2} \alpha_{2}=C \sin (2 \psi) \alpha_{1}-(C \cos (2 \psi)+1) \alpha_{2} \\
= & C \sin (2 \psi)\left(\cos (2 \psi) \vartheta_{1}-\sin (2 \psi) \vartheta_{2}\right) \\
& -(C \cos (2 \psi)+1)\left(\sin (2 \psi) \vartheta_{1}+\cos (2 \psi) \vartheta_{2}\right) \\
= & -\sin (2 \psi) \vartheta_{1}-(C+\cos (2 \psi)) \vartheta_{2} .
\end{aligned}
$$

In the interests of completeness, it is important to verify the following Theorem.

Theorem 14. The function B satisfies (57) provided $\psi$ satisfies both (58) and (64).

Proof. Differentiating $B_{1}$ and $B_{2}$ given by (86), the left side of (57) is found to be

$$
\begin{aligned}
B_{21}- & B_{12}+B_{2} \\
= & 2 B_{1} \psi_{1}+B_{2}\left(2 \psi_{2}+1\right)+2 B\left(\psi_{11}+\psi_{22}+\psi_{1}\right) \\
& +A^{-2}\left((a c)_{1} \sin (2 \psi)-(a c)_{2} \sin (2 \psi)\right) \\
& -2 a c B A^{-2}(\cos (2 \psi) \sin (2 \psi)-\sin (2 \psi) \cos (2 \psi)) \\
& +\left(1+a c A^{-2}\right)\left(2 \psi_{1} \cos (2 \psi)+\left(2 \psi_{2}+1\right) \sin (2 \psi)\right) \\
= & 2\left(1+a c A^{-2}\right)\left(2 \psi_{1} \cos (2 \psi)+\left(2 \psi_{2}+1\right) \sin (2 \psi)\right) \\
& +A^{-2}\left((a c)_{1} \sin (2 \psi)-(a c)_{2} \cos (2 \psi)\right)
\end{aligned}
$$

To simplify this, (58) has been substituted. Using (75) and $* d(a c)=(a c)_{1} \alpha_{2}-(a c)_{2} \alpha_{1}$, it follows that

$$
* d(a c) \wedge \vartheta_{2}=\left((a c)_{1} \sin (2 \psi)-(a c)_{2} \cos (2 \psi)\right) \alpha_{1} \wedge \alpha_{2} \text {. }
$$

Note that the coefficient of $\alpha_{1} \wedge \alpha_{2}$ in this appears in the compatibility condition. To express it in another way, begin by finding the exterior derivative of $4 a c=(a+c)^{2}-(a-c)^{2}$,

$$
4 d(a c)=2(a+c)(a-c) \vartheta_{1}-2(a-c)^{2}\left(\alpha_{1}+2 * \omega_{12}\right) .
$$

Applying the Hodge operator to both sides of this gives, upon rearranging terms,

$$
2 * \frac{d(a c)}{a-c}=(a+c) \vartheta_{2}-(a-c)\left(\alpha_{2}-2 \omega_{12}\right) .
$$

Consequently, we can write

$$
\begin{aligned}
& -\frac{2}{(a-c)^{2}} * d(a c) \wedge \vartheta_{2} \\
& \quad=\left(\alpha_{2}-2 \omega_{12}\right) \wedge \vartheta_{2} \\
& \quad=-\left(2 \psi_{1} \cos (2 \psi)+\left(2 \psi_{2}+1\right) \sin (2 \psi)\right) \alpha_{1} \wedge \alpha_{2} .
\end{aligned}
$$

Therefore, it must be that

$$
\begin{aligned}
& -(a c)_{1} \sin (2 \psi)+(a c)_{2} \cos (2 \psi) \\
& =-\frac{1}{2}(a-c)^{2}\left(2 \psi_{1} \cos (2 \psi)+\left(2 \psi_{2}+1\right) \sin (2 \psi)\right) .
\end{aligned}
$$

It follows that when $f=B$, (57) finally reduces to the form

$$
\left(1+H^{2} A^{-2}\right)\left[2 \psi_{1} \cos (2 \psi)+\left(2 \psi_{2}+1\right) \sin (2 \psi)\right]=0 .
$$

The first factor is clearly nonzero, so the second factor must vanish. This of course is equivalent to the constraint (64).

\section{Intrinsic Characterization of $M$}

During the prolongation of the exterior differential system, the additional variables $\psi, A, B$, and $C$ have been introduced. The significance of the appearance of the function $C$, is that the process terminates and the differentials of all these functions can be computed without the need to introduce more functions. This means that the exterior differential system has finally closed.

The results of the previous section, in particular the lemmas, can be collected such that they justify the following.

Proposition 15. The differential system generated in terms of the differentials of the variables $\psi, A, B$, and $C$ is closed. The variables $H, J, A, B$, and $C$ remain constant along the $\vartheta_{2}$-curves so $\vartheta_{1}=0$. Hence, an isometry that preserves $H$ must map the $\vartheta_{1}, \vartheta_{2}$ curves onto the corresponding $\vartheta_{1}^{*}, \vartheta_{2}^{*}$ curves of the associated surface $M^{*}$ which is isometric to $M$.

Along the $\vartheta_{1}, \vartheta_{2}$ curves, consider the normalized frame,

$$
\begin{aligned}
& \zeta_{1}=\cos (\psi) e_{1}+\sin (\psi) e_{2}, \\
& \zeta_{2}=-\sin (\psi) e_{1}+\cos (\psi) e_{2} .
\end{aligned}
$$


The corresponding coframe and connection form are

$$
\begin{aligned}
\xi_{1} & =\cos (\psi) \omega_{1}+\sin (\psi) \omega_{2}, \\
\xi_{2} & =-\sin (\psi) \omega_{1}+\cos (\psi) \omega_{2}, \\
\xi_{12} & =d \psi+\omega_{12} .
\end{aligned}
$$

Then $\vartheta_{1}$ can be expressed as a multiple of $\xi_{1}$ and $\vartheta_{2}, \vartheta_{12}$ in terms of $\xi_{2}$, and the differential system can be summarized here:

$$
\begin{gathered}
\vartheta_{1}=A \xi_{1}, \quad \vartheta_{2}=A \xi_{2}, \\
\vartheta_{12}=\xi_{12}+* d \log A=-C A \xi_{2}, \\
d \log A=A B \xi_{1}, \quad d B=A\left(B C+1+a c A^{-2}\right) \xi_{1}, \\
d C=A\left(C^{2}-1\right) \xi_{1}, \\
d H=A J \xi_{1}, \quad d J=A J(2 B+C) \xi_{1} .
\end{gathered}
$$

The condition $d \vartheta_{1}=0$ is equivalent to

$$
d A \wedge \xi_{1}+A d \xi_{1}=0 .
$$

This implies that $d \xi_{1}=0$ since $d A$ is proportional to $\xi_{1}$. Also $d * \vartheta_{12}=0$ is equivalent to $d * \xi_{12}=0$.

Moreover, $d * \xi_{12}=0$ is equivalent to the fact that the $\xi_{1}$, $\xi_{2}$-curves can be regarded as coordinate curves parameterized by isothermal parameters. Therefore, along the $\xi_{1}, \xi_{2}$-curves, orthogonal isothermal coordinates denoted $(s, t)$ can be introduced. The first fundamental form of $M$ then takes the form,

$$
I=\xi_{1}^{2}+\xi_{2}^{2}=E(s)\left(d s^{2}+d t^{2}\right) .
$$

Now suppose we set $e(s)=\sqrt{E(s)}$, then

$$
\begin{gathered}
\xi_{1}=e(s) d s, \quad \xi_{2}=e(s) d t, \\
\xi_{12}=\frac{e^{\prime}(s)}{e^{2}(s)} \xi_{2}=\frac{e^{\prime}(s)}{e(s)} d t .
\end{gathered}
$$

This means such a surface is isometric to a surface of revolution. Since $\psi, d * \xi_{12}=0$, (100) implies that $d * \omega_{12}=0$. This can be stated otherwise as the principal coordinates are isothermal and so $M$ is an isothermic surface.

Since $A, B, C, H$, and $J$ are functions of only the variable $s$, this implies that $H$ and $J$ or $H$ and $K$ are constant along the t-curves where $s$ is constant. This leads to the following proposition.

\section{Proposition 16.}

$$
d H \wedge d K=0, \quad \xi_{12}=-(C+B) A \xi_{2} .
$$

This is equivalent to the statement that $M$ is a Weingarten surface.

Proof. The first result follows from the statement about the coordinate system above. Since $\vartheta_{12}=\xi_{12}+* d \log A=-C A \xi_{2}$ and $d A=A^{2} B \xi_{1}$,

$$
\begin{aligned}
\xi_{12} & =-C A \xi_{2}-* d \log A=-C A \xi_{2}-* A^{-1} d A \\
& =-C A \xi_{2}-A B * \xi_{1}=-(C+B) A \xi_{2} .
\end{aligned}
$$

Consequently, the geodesic curvature of each $\xi_{2}$-curve, $s$ constant is

$$
\frac{e^{\prime}(s)}{e^{2}(s)}=-A(B+C),
$$

which is constant.

To express the $\omega_{i}$ in terms of $d s$ and $d t$, start by writing $\omega_{i}$ in terms of the $\xi_{i}$ and then substituting (104),

$$
\begin{aligned}
& \omega_{1}=\cos (\psi) e d s-\sin (\psi) e d t, \\
& \omega_{2}=\sin (\psi) e d s+\cos (\psi) e d t .
\end{aligned}
$$

Subscripts $(s, t)$ denote differentiation and $H_{s}=H^{\prime}$ is used interchangeably. Beginning with $d H=H^{\prime} d s$ and using (108), we have

$$
\begin{aligned}
d H= & H_{1} \omega_{1}+H_{2} \omega_{2}=\left(H_{1} \cos (\psi)+H_{2} \sin (\psi)\right) e d s \\
& +\left(-H_{1} \sin (\psi)+H_{2} \cos (\psi)\right) e d t=H^{\prime} d s .
\end{aligned}
$$

Equating coefficients of differentials, this implies that

$$
\begin{gathered}
H_{1} e \cos (\psi)+H_{2} e \sin (\psi)=H^{\prime}, \\
-H_{1} \sin (\psi)+H_{2} \cos (\psi)=0 .
\end{gathered}
$$

Solving this as a linear system we obtain $H_{1}, H_{2}$,

$$
H_{1}=\frac{H^{\prime}}{e} \cos (\psi), \quad H_{2}=\frac{H^{\prime}}{e} \sin (\psi) .
$$

Noting that $u=H_{1} / J$ and $v=H_{2} / J$, using (100) the forms $\alpha_{i}$ can be expressed in terms of $d s, d t$ :

$$
\begin{aligned}
& \alpha_{1}=\frac{H^{\prime}}{J}(\cos (2 \psi) d s-\sin (2 \psi) d t), \\
& \alpha_{2}=\frac{H^{\prime}}{J}(\sin (2 \psi) d s+\cos (2 \psi) d t) .
\end{aligned}
$$

Substituting $\xi_{1}$ from (104) into $d H=A J \xi_{1}$,

$$
d H=H^{\prime} d s=A J \xi_{1}=A J e(s) d s .
$$

Therefore, $H^{\prime}=A J e>0$ and so $H(s)$ is an increasing function of $s$. Now define the function $Q(s)$ to be

$$
Q=\frac{H^{\prime}}{J}=A \cdot e>0 .
$$

Substituting (114) into (112), the $\alpha_{i}$ are expressed in terms of $Q$ as well. The equations (30) in Theorem 5 can easily be expressed in terms of $\psi$ and $Q$.

Theorem 17. Equations (30) are equivalent to the following system of coupled equations in $\psi$ and $Q$ :

$$
\begin{aligned}
& \sin (2 \psi)(\log (Q))_{s}+2 \cos (2 \psi) \psi_{s}-2 \sin (2 \psi) \psi_{t}=0 \\
& \cos (2 \psi)(\log (Q))_{s}-2 \sin (2 \psi) \psi_{s}-2 \cos (2 \psi) \psi_{t}=Q .
\end{aligned}
$$


Moreover, (115) are equivalent to the following first-order system:

$$
\psi_{s}=-\frac{1}{2} Q \sin (2 \psi), \quad \psi_{t}=\frac{1}{2}(\log (Q))_{s}-\frac{1}{2} Q \cos (2 \psi) .
$$

System (116) can be thought of as a type of Lax pair. Moreover, (116) implies that $\psi$ is harmonic as well. Differentiating $\psi_{s}$ with respect to $s$ and $\psi_{t}$ with respect to $t$, it is clear that $\psi$ satisfies Laplace's equation in the $(s, t)$ variables $\psi_{s s}+\psi_{t t}=0$. This is another proof that $\psi$ is harmonic.

Theorem 18. The function $Q(s)$ satisfies the following secondorder nonlinear differential equation:

$$
Q^{\prime \prime}(s) Q(s)-\left(Q^{\prime}(s)\right)^{2}=Q^{4}(s) \text {. }
$$

There exists a first integral for this equation of the following form:

$$
Q^{\prime}(s)^{2}=Q(s)^{4}+\kappa Q(s)^{2}, \quad \kappa \in \mathbb{R}
$$

Proof. Equation (117) is just the compatibility condition for the first-order system (116). The required derivatives are

$$
\begin{aligned}
& \psi_{s t}=-\frac{Q}{2} \cos (2 \psi)\left((\log Q)_{s}-Q \cos (2 \psi)\right), \\
& \psi_{t s}=\frac{1}{2}(\log Q)_{s s}-\frac{1}{2} Q_{s} \cos (2 \psi)+Q \sin (2 \psi) \psi_{s} .
\end{aligned}
$$

Equating derivatives $\psi_{s t}=\psi_{t s}$, the required (117) follows.

Differentiating both sides of (118) we get

$$
Q^{\prime \prime}(s)=2 Q(s)^{3}+\kappa Q(s) .
$$

Isolating $\kappa Q(s)$ from (118) and substituting it into (120), (117) appears.

It is important to note that the function $C$ which appears when the differential ideal closes can be related to the function $Q$.

\section{Corollary 19.}

$$
C=\left(\frac{1}{Q}\right)^{\prime}
$$

Proof. Using $\vartheta_{i}$ from (101) in Lemma 13, in the $s, t$ coordinates

$$
\begin{aligned}
2 d \psi & =-\sin (2 \psi) \text { Aeds }-(C+\cos (2 \psi)) \text { Aedt } \\
& =\psi_{s} d s+\psi_{t} d t .
\end{aligned}
$$

Hence using (116), this implies that $2 \psi_{s}=-\sin (2 \psi) A e=$ $-Q \sin (2 \psi)$, hence $Q=A e$. The second equation in (116) for $\psi_{t}$ implies that $(C+\cos (2 \psi)) A e=Q \cos (2 \psi)-(\log Q)^{\prime}$. Replacing $A e=Q$, this equation simplifies to the form (121).

\section{Integrating the Lax Pair System}

It is clear that the first order equation in (116) for $Q(s)$ is separable and can be integrated. The integral depends on whether $K$ is zero or nonzero:

$$
\begin{gathered}
Q(s)=\frac{1}{\epsilon s+\gamma}, \quad K=0 \\
\log \left(\frac{2\left(K+\sqrt{K} \sqrt{Q^{2}+K}\right)}{Q}\right)=\epsilon \sqrt{K} s+\gamma, \quad K \neq 0 .
\end{gathered}
$$

Here, $\epsilon= \pm 1$ and $\gamma$ is the last constant of integration. Taking specific choices for the constants, for example, $e^{\gamma}=2 \sqrt{K}$ when $K \neq 0$ and $a=\sqrt{K}$, the set of solutions (123) for $Q(s)$ can be summarized below:

$$
\begin{array}{cccc}
\operatorname{Dom}(s) & Q(s) & \operatorname{Dom}(s) & Q(s) \\
s>0 & \frac{1}{s} & s<0 & -\frac{1}{s} \\
0<s<\frac{\pi}{a} & \frac{a}{\sin (a s)} & -\frac{\pi}{a}<s<0 & -\frac{a}{\sin (a s)} \\
s>0 & \frac{a}{\sinh (a s)} & s<0 & -\frac{a}{\sinh (a s)} .
\end{array}
$$

It is presumed that other choices of the constants can be geometrically eliminated in favor of (124). The solutions (124) are then substituted back into linear system (116). The first equation in (116) implies that either of the two cases

$$
\psi \equiv 0, \quad \bmod \frac{\pi}{2} ; \quad \frac{2 \psi_{s}}{\sin (2 \psi)}=-\mathrm{Q} .
$$

Substitute $\psi \equiv 0$ into the second equation in (116). It implies that $(\log Q)_{s}=Q$ and $\psi=\pi / 2$ give $(\log Q)_{s}=-Q$. In both cases $Q(s)$ is a solution which already appears in (124).

For the second case in (125), the equation can be put in the form

$$
(\log |\tan (\psi)|)_{s}=-Q
$$

Integrating we have for some function $y(t)$ to be determined,

$$
\tan (\psi)=e^{-\int Q(s) d s} \cdot y(t) .
$$

Therefore, $\tan (\psi)$ can be obtained by substituting for $Q(s)$ for each of the three cases in (124). The upper sign holds for $s>0$ and the lower sign holds if $s<0$.

(i) $Q(s)= \pm s^{-1},-\int Q(s) d s=\log |s|^{\mp}$, and

$$
\tan (\psi)=s^{\mp} \cdot y(t) .
$$

(ii) $Q(s)= \pm(a / \sin (a s)),-\int Q(s) d s=\log \mid \csc (a s)-$ $\left.\cot (a s)\right|^{\mp}$, and

$$
\tan (\psi)=\left(\tan \left(\frac{a s}{2}\right)\right)^{\mp} \cdot y(t) .
$$


(iii) $Q(s)= \pm(a / \sinh (a s)),-\int Q(s) d s=\mp \operatorname{arctanh}\left(e^{a s}\right)$, and

$$
\tan (\psi)=\left(\tanh \left(\frac{a s}{2}\right)\right)^{\mp} \cdot y(t)
$$

In case (ii), if $s>0$ and $y(t)= \pm 1$ then $\psi= \pm(1 / 2)(a s+$ $\pi), \bmod \pi$, and if $s<0$ and $y(t)= \pm 1$, then $\psi= \pm(1 / 2) a s$, $\bmod \pi$.

It remains to integrate the second equation of the Lax pair (116) using solutions for both $Q(s)$ and $\tan (\psi)$. The first case (i) is not hard and will be shown explicitly here. The others can be done, and more complicated cases are considered in the Appendix.

(i) Consider $Q(s)=s^{-1}$ and $\tan (\psi)=s^{-1} \cdot y(t)$. The second equation in (116) simplifies considerably to $y_{t}=-1$; therefore,

$$
y(t)=-(t+\sigma), \quad \tan (\psi)=-\frac{(t+\sigma)}{s} .
$$

For $Q(s)=-s^{-1}$ and $\tan (\psi)=s \cdot y(t)$, the second equation of (116) becomes $y_{t}=-y^{2}$; therefore,

$$
y(t)=\frac{1}{t+\sigma}, \quad \tan (\psi)=\frac{s}{t+\sigma} .
$$

\section{A Third Order Equation for $H$ and Fundamental Forms}

Since $\xi_{12}=(\log e(s))^{\prime} d t$, using (104) $\omega_{12}$ can be written as

$$
\omega_{12}=\xi_{12}-d \psi=(\log e(s))^{\prime} d t-d \psi .
$$

Using (14) and (112) for $\alpha_{1}$, it follows that

$$
\begin{aligned}
d \log (J)= & Q(\cos (2 \psi) d s-\sin (2 \psi) d t) \\
& -2 *\left(\psi_{t} d t+\psi_{s} d s\right)+2 *(\log (e(s)))^{\prime} d t .
\end{aligned}
$$

When the $\omega_{i}$ are put in the $s, t$ coordinates, using $* \omega_{1}=\omega_{2}$, it can be stated that $* d s=d t$ and $* d t=-d s$. Consequently, $d \log (J)$ simplifies to

$$
\begin{aligned}
d \log (J)= & \left(Q \cos (2 \psi)+2 \psi_{t}-2(\log (e(s)))^{\prime}\right) d s \\
& +\left(-Q \sin (2 \psi)-2 \psi_{s}\right) d t .
\end{aligned}
$$

First order system (116) permits this to be written using $e(s)=$ $\sqrt{E(s)}$ as

$$
(\log (J))^{\prime}+(\log (E))^{\prime}=(\log (Q))^{\prime} .
$$

Hence there exists a constant $\tau$ independent of $s$ such that $E \cdot J=\tau Q$ or

$$
E=\tau \frac{Q}{J}=\tau \frac{Q^{2}}{H^{\prime}} .
$$

This result (137) for $E$ is substituted into the Gauss equation $-\left((\log (E))_{s s}+(\log (E))_{t t}\right)=2 E\left(H^{2}-J^{2}\right)$ giving

$$
(\log (E))^{\prime \prime}=2(\log (Q))^{\prime \prime}-\left(\log \left(H_{s}\right)\right)^{\prime \prime}=2 Q^{2}-\left(\frac{H^{\prime \prime}}{H^{\prime}}\right)^{\prime} .
$$

Therefore, the Gauss equation transforms into a third-order differential equation in the $s$ variable,

$$
\left(\frac{H^{\prime \prime}}{H^{\prime}}\right)^{\prime}+2 \tau H=2 Q^{2}\left(1+\tau \frac{H^{2}}{H^{\prime}}\right)
$$

Thus a characterization of Bonnet surfaces is reached by means of the solutions to these equations. This equation determines the function $H(s)$ and after that the functions $J(s)$ and $E(s)$. Therefore, Bonnet surfaces have as first fundamental form the expression

$$
I=E(s)\left(d s^{2}+d t^{2}\right), \quad E(s)=\tau \frac{Q^{2}(s)}{H^{\prime}(s)} .
$$

Since $\psi$ is the angle from the principal axis $e_{1}$ to the $s$-curve with $t$ equals constant, the second fundamental form is given by

$$
I I=L d s^{2}+2 M d s d t+N d t^{2},
$$

where the coefficients $L, M$, and $N$ are given by

$$
\begin{aligned}
L & =E(H+J \cos (2 \psi))=E H+\tau Q \cos (2 \psi), \\
M & =-E J \sin (2 \psi)=-\tau Q \sin (2 \psi), \\
N & =E(H-J \cos (2 \psi)) .
\end{aligned}
$$

\section{Appendix}

It is worth seeing how the second equation in (116) can be integrated for cases (ii) and (iii). Only the case $s>0$ will be done with $Q(s)$ taken from (124).

(a) Differentiating $\tan (\psi)$ given in (129), we obtain that

$$
\psi_{t}=\frac{\tan (a s / 2)}{\tan ^{2}(a s / 2)+y^{2}} y_{t}(t)
$$

The following identities are required to simplify the result:

$$
\begin{aligned}
\tan (a s) & =\frac{2 \tan (a s / 2)}{1-\tan ^{2}(a s / 2)}, \\
\cos (2 \psi) & =\frac{\tan ^{2}(\text { as } / 2)-y^{2}}{\tan ^{2}(a s / 2)+y^{2}} .
\end{aligned}
$$

Substituting $\psi_{t}$ into (116), we obtain

$$
\begin{aligned}
& \frac{2 \tan (a s / 2)}{\tan ^{2}(a s / 2)+y^{2}} y_{t} \\
& \quad=-a \cot (a s)-\frac{a}{\sin (a s)} \frac{\tan ^{2}(a s / 2)-y^{2}}{\tan ^{2}(a s / 2)+y^{2}} .
\end{aligned}
$$

Simplifying this, we get

$$
\begin{aligned}
\frac{4}{a} y_{t}= & -\frac{1}{2}\left(1-\tan ^{2}\left(\frac{a s}{2}\right)\right)-\frac{1}{2}\left(\cot ^{2}\left(\frac{a s}{2}\right)-1\right) y^{2} \\
& -\sec ^{2}\left(\frac{a s}{2}\right)+\csc ^{2}\left(\frac{a s}{2}\right) y^{2} .
\end{aligned}
$$


This simplifies to the elementary equation,

$$
y_{t}=\frac{a}{2}\left(y^{2}-1\right), \quad y(t)=-\tanh \left(\frac{a t}{2}+\eta\right) .
$$

Here, $\eta$ is an integration constant. To summarize then,

$$
\tan (\psi)=\tanh \left(\frac{a t}{2}+\eta\right) \cdot \tan \left(\frac{a s+\pi}{2}\right) .
$$

(b) Consider now $s>0$ and take $Q(s)$ from the last line of (124). Differentiating $\tan (\psi)$ from (130), we get

$$
\psi_{t}=\frac{\operatorname{coth}(a s / 2)}{1+\operatorname{coth}^{2}(a s / 2) y^{2}} y_{t}(t) .
$$

In this case, the following identities are needed:

$$
\begin{gathered}
\tanh (a s)=\frac{2 \tanh (a s / 2)}{1+\tanh ^{2}(a s / 2)}, \\
\cos (2 \psi)=\frac{1-\operatorname{coth}^{2}(a s / 2) y^{2}}{1+\operatorname{coth}^{2}(a s / 2) y^{2}} .
\end{gathered}
$$

Therefore, (116) becomes

$$
\begin{aligned}
& 2 \frac{\operatorname{coth}(a s / 2)}{1+\operatorname{coth}^{2}(a s / 2) y^{2}} y_{t} \\
& \quad=-a \operatorname{coth}(a s)-\frac{a}{\sinh (a s)} \frac{\tanh ^{2}(a s / 2)-y^{2}}{\tanh ^{2}(a s / 2)+y^{2}} .
\end{aligned}
$$

This reduces to

$$
\begin{aligned}
-\frac{4}{a} y_{t}= & \left(1+\tanh ^{2}\left(\frac{a s}{2}\right)+\operatorname{sech}^{2}\left(\frac{a s}{2}\right)\right) \\
& +\left(\operatorname{coth}^{2}\left(\frac{a s}{2}\right)+1-\operatorname{csch}^{2}\left(\frac{a s}{2}\right)\right) y^{2} .
\end{aligned}
$$

Simplifying and integrating, it has been found that

$$
y_{t}=-\frac{a}{2}\left(1+y^{2}\right), \quad y(t)=-\tan \left(\frac{a t}{2}+\eta\right) .
$$

To summarize then, it has been shown that

$$
\tan (\psi)=\cot \left(\frac{a t}{2}+\eta\right) \cdot \operatorname{coth}\left(\frac{a s}{2}\right) .
$$

These results apply to the case $s>0$ and similar results can be found for the case $s<0$ as well.

\section{Conflict of Interests}

The author declares that there is no conflict of interests regarding the publication of this paper.

\section{References}

[1] A. I. Bobenko and U. Eitner, Painlevé Equations in the Differential Geometry of Surfaces, vol. 1753 of Lecture Notes in Mathematics, Springer, Berlin, Germany, 2000.
[2] G. Darboux, Théorie des Surfaces, Partie 3, Paris, France, 1894.

[3] S. S. Chern, "Surface theory with Darboux and Bianchi", in Miscellanea Mathematica, pp. 59-69, Springer, Berlin, Germany, 1991.

[4] H. Hopf, Differential Geometry in the Large, Part II, vol. 1000 of Lecture Notes in Mathematics, Springer, 1983.

[5] P. O. Bonnet, "Mémoire sur la theorie des surface applicables sur une surface donneé," Journal de l'École Polytechnique-Paris, vol. 42, Cahier, pp. 72-92, 1867.

[6] S. S. Chern, "Deformations of Surfaces preserving principal curvatures," in Differential Geometry and Complex Analysis, $\mathrm{H}$. E. Rauch, Ed., pp. 155-163, Springer, Berlin, Germany, 1985.

[7] I. M. Roussos and G. E. Hernandez, "On the number of distinct isometric immersions of a Riemannian surface into $R^{3}$ with given mean curvature," American Journal of Mathematics, vol. 112, no. 1, pp. 71-85, 1990.

[8] I. M. Roussos, "Global results on Bonnet surfaces," Journal of Geometry, vol. 65, no. 1-2, pp. 151-168, 1999.

[9] J. Lawson and R. d. A. Tribuzy, "On the mean curvature function for compact surfaces," Journal of Differential Geometry, vol. 16, no. 2, pp. 179-183, 1981.

[10] A. G. Colares and K. Kenmotsu, "Isometric deformations of surfaces in $R^{3}$ preserving the mean curvature function," Pacific Journal of Mathematics, vol. 136, no. 1, pp. 71-80, 1989.

[11] X. Chen and C. K. Peng, "Deformation of surfaces preserving principal curvatures," in Differential Geometry and Topology, vol. 1369 of Lecture Notes in Mathematics, pp. 63-73, Springer, Berlin, Germany, 1987.

[12] S. S. Chern, W. H. Chen, and K. S. Lam, Lectures in Differential Geometry, vol. 1 of Series on University Mathematics, World Scientific, Singapore, 1999.

[13] K. Kenmotsu, "An intrinsic characterization of $H$-deformable surfaces," Journal of the London Mathematical Society, vol. 49, no. 3, pp. 555-568, 1994.

[14] P. Bracken, "Cartan's theory of moving frames and an application to a theorem of Bonnet," Tensor, vol. 70, no. 3, pp. 261-274, 2008. 


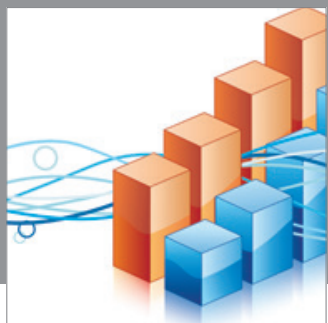

Advances in

Operations Research

mansans

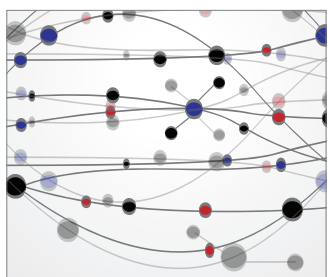

The Scientific World Journal
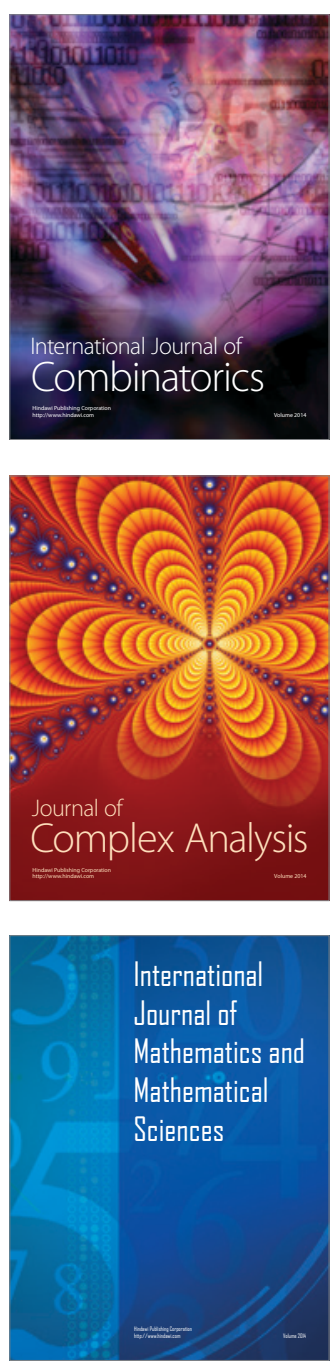
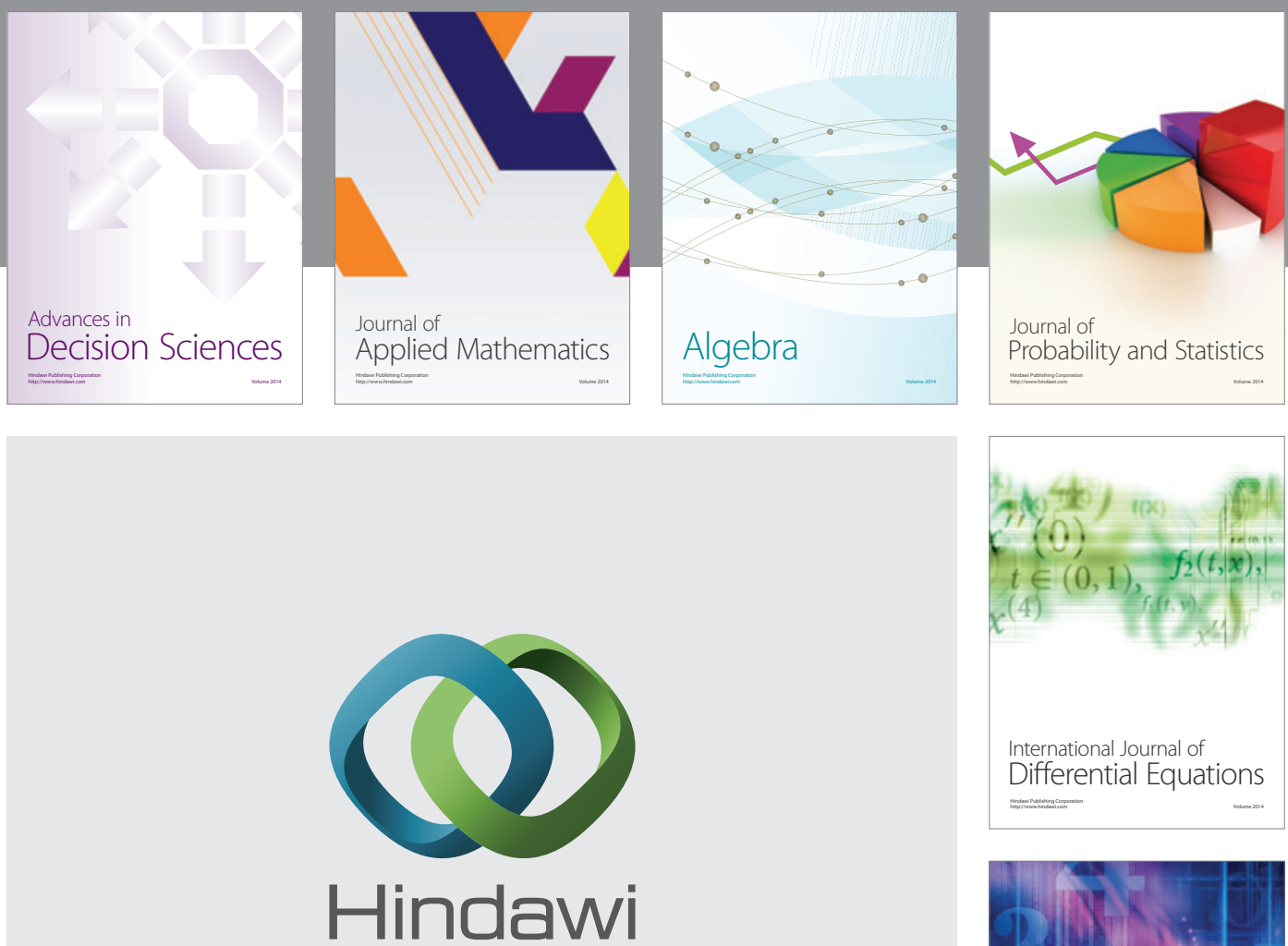

Submit your manuscripts at http://www.hindawi.com
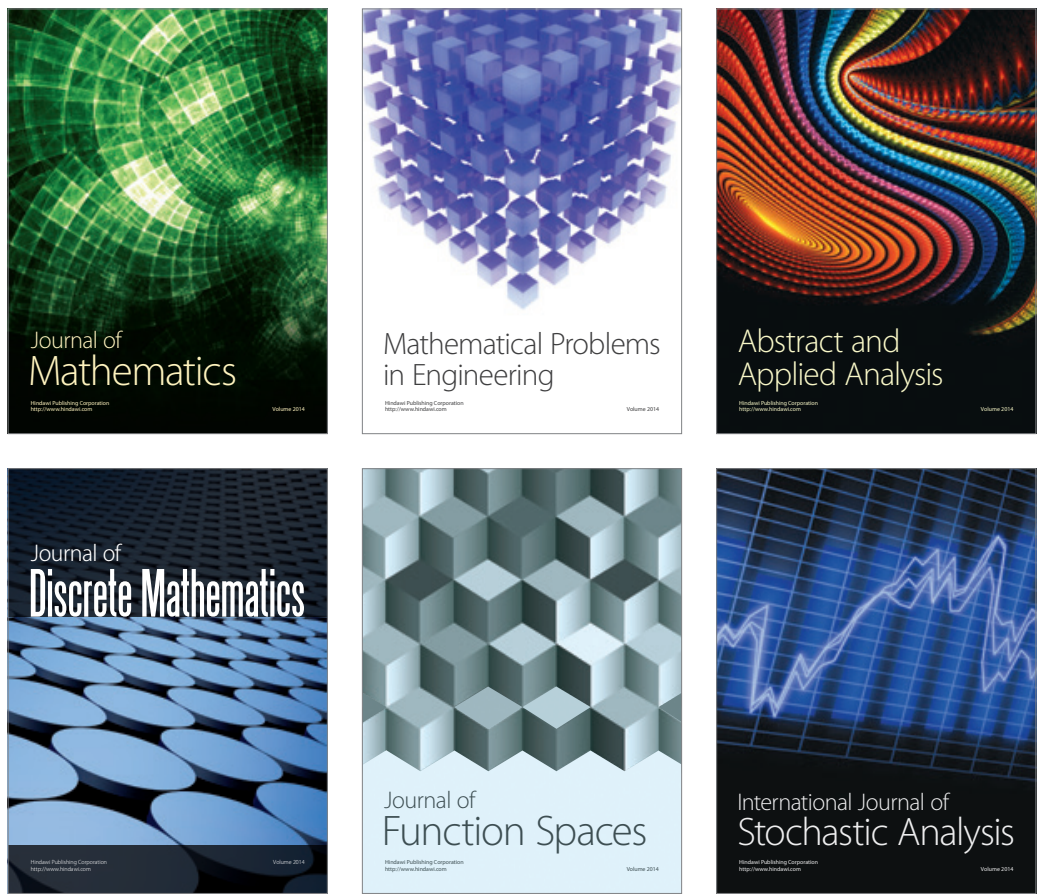

Journal of

Function Spaces

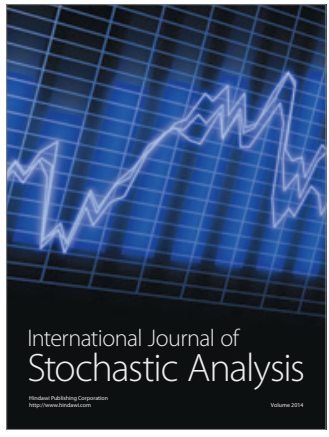

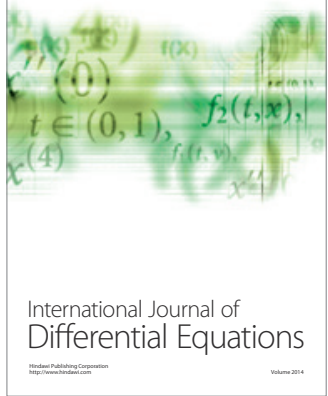
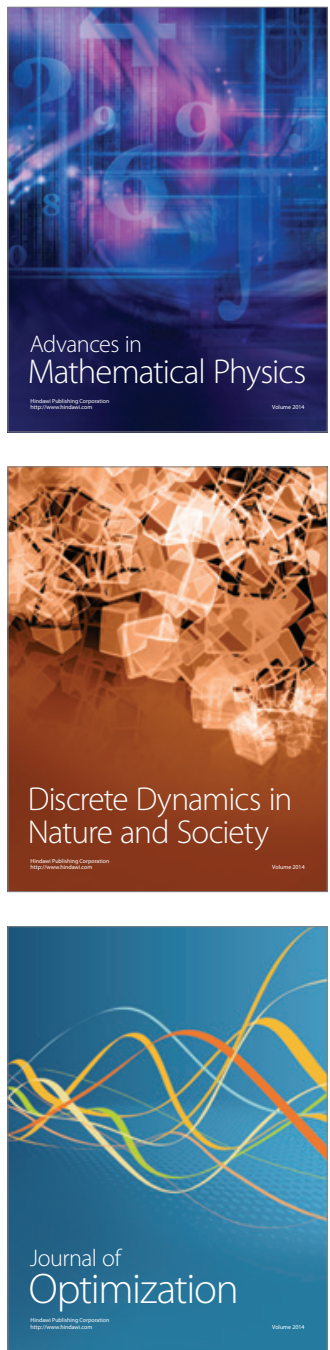